\title{
Magenverkleinerung senkt das Schlaganfallrisiko bei Adipösen
}

In den meisten epidemiologischen Studien ist Übergewicht ein Risikofaktor für kardiovaskuläre Ereignisse einschließlich Herzinfarkt und Schlaganfall. Allerdings gibt es nur relativ wenige Studien, die systematisch den Einfluss einer Gewichtsreduktion auf das Risiko vaskulärer Ereignisse untersucht haben, und diese haben zu widersprüchlichen Ergebnissen geführt.

- Operative Eingriffe zur Verkleinerung des Volumens des Magens sind seit vielen Jahren bei schwer übergewichtigen Patienten etabliert und führen in aller Regel zu einer signifikanten bis dramatischen Reduktion des Körpergewichts. Daher bietet es sich an, den Effekt dieser Operation auf kardiovaskuläre Ereignisse zu untersuchen.

Es handelt sich um eine noch laufende, nicht randomisierte, prospektive, kontrollierte Studie in Schweden, in die zwischen 1987 und 2001 insgesamt 4047 Patienten mit Übergewicht eingeschlossen wurden.

Einschlusskriterien für die Operation waren ein Alter zwischen 37 und 60 Jah- ren und ein Body-Mass-Index (BMI) von mindestens 34 für Männer und 38 für Frauen. Nach im Mittel 14,7 Jahren hatten die operierten Patienten ihr Körpergewicht um $18 \%$ reduziert, während es in der Kontrollgruppe unverändert war. Es kam zu 49 kardiovaskulären Todesfällen bei den 2037 Patienten in der Kontrollgruppe und zu 28 kardiovaskulären Todesfällen bei den 2010 Patienten in der Operationsgruppe. Dies entspricht einer relativen Risikoreduktion von $44 \%$. Nach Korrektur für Risikofaktoren reduzierte die Operation sowohl das Risiko von tödlichen Schlaganfällen um 66\% und nicht tödlichen Schlaganfällen um $44 \%$.

\section{Kommentar}

Die Ergebnisse dieser Studie sind in mehrfacher Hinsicht sehr relevant. Die Studie zeigt, dass eine deutliche Gewichtsreduktion um durchschnittlich 20\% zu einer signifikanten und klinisch relevanten Reduktion kardiovaskulärer und zerebrovaskulärer Ereignisse führt. Die Studie zeigt aber auch eindrucksvoll, dass die üblichen Maßnahmen zur Gewichtsreduktion wie Beratung und Diät offenbar völlig wirkungslos sind. Dies sollte Anlass sein, bei deutlich übergewichtigen Personen, die erfolglos Diäten und Sport zur Gewichtsreduktion eingesetzt haben und motiviert sind, in Einzelfällen entsprechende operative Eingriffe durchzuführen.

H.-C. DIENER —

- L. Sjöström et al.

Bariatric surgery and long-term cardiovascular events. JAMA307 (2012) 56-65

\section{Abdominelle Verkalkungen}

\section{Eine 63-jährige Frau, die seit 30 Jahren in einem Schweinemastbe- trieb arbeitete, wurde wegen Ver- schlechterung ihrer seit 30 Jahren bekannten Epilepsie vorgestellt.}

— Vor 25 Jahren hatte sich die Patientin wegen schmerzloser subkutaner Knötchen einer Hautbiopsie unterzogen, welche die Diagnose einer Zystizerkose ergab. Sie war damals zehn Tage lang mit Praziquantel behandelt worden. Die körperliche Untersuchung war unauffällig, ein Tuberkulintest negativ. Abdominelle Symptome wurden nicht angegeben. Im CT des Gehirns zeigten sich zahlreiche fokale Verkalkungen in beiden Hemisphären. Auch in der Abdomen-Übersicht wurden multiple, unregelmäßig begrenzte Verkalkungen festgestellt, die über das ganze Abdomen diffus verstreut lagen (Abb.). Wegen der hohen Kosten und aus Angst vor Komplikationen verweigerte die Patientin eine diagnostische Laparoskopie. Aufgrund der anamnestisch bekannten Zystizerkose und der übrigen Untersu-
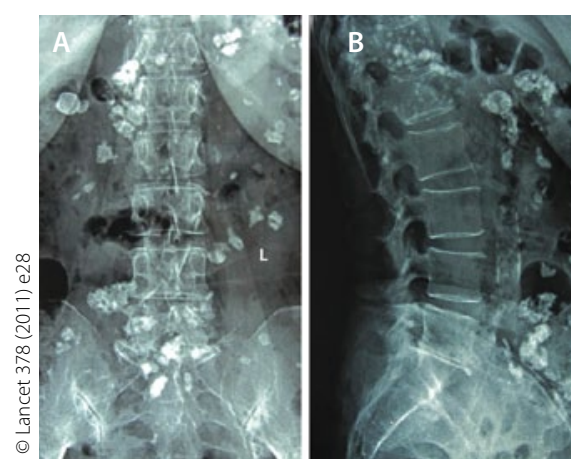

Abdomen-Übersichtsaufnahme a.p. (A) und seitlich (B) mit multiplen, unregelmäßig begrenzten Verkalkungen. chungsbefunde vermutete man als Ursache der multiplen abdominellen Verkalkungen die Zystizerkose. Unter Therapie mit Carbamazepin besserte sich die Epilepsie der Patientin.

\section{Kommentar}

Die Zystizerkose ist eine systemische parasitäre Infektion, die durch den Schweinebandwurm hervorgerufen wird. Verkalkungen im Bereich des Gehirns kommen bei dieser Erkrankung häufig vor, ausgedehnte abdominelle Verkalkungen sind jedoch eine Rarität und wurden bisher kaum beobachtet. Es handelt sich um einen nicht aktiven Zustand, der keiner weiteren Behandlung bedarf.

H. S. FÜESSL =

\section{-W. Zhao, F. Huang}

(Korres.: zhaowei-1@medmail.com.cn): Abdominal calcifications. Lancet 378 (2011) e28 